\title{
EFFECT OF SOME ORGANIC FERTILIZERS FROM DIFFERENT SOURCES ON YIELD AND QUALITY OF THOMPSON SEEDLESS GRAPEVINES (Vitis vinifera, L.) Mostafa, M.F.M.*; A. Hegazi ${ }^{\star}$; M.M. El-Mogy ${ }^{\star \star}$ and B.E.A. Belal ${ }^{\star *}$ * Pomology Department, Faculty of Agriculture, Mansoura University ${ }^{* *}$ Horticulture Research Institute, Agriculture Research Center
}

\begin{abstract}
The present study was carried out during 2003, 2004 and 2005 seasons on 15 years old Thompson seedless grapevines grown on a clay soil in a private vineyard at EL-Tawela village near Mansoura city, Dakahlia governorate. The aim of the present study was to investigate the effect of addition different sources of organic fertilizers as a partial substitute for nitrogen mineral fertilizers and their effect on bud behaviour, growth, mineral content of the leaves, yield and quality of Thompson Seedless grapevines. Farmyard manure (FYM), rice straw compost (RSC), Damitta town refuses (DTR) and Mansoura town refuses (MTR) were used with mineral nitrogen source (Ammonium sulphate) during this investigation. All vines were received the same nitrogen requirements ( 80 units of nitrogen per fed), but with replacement of 25,50 and $75 \%$ of the nitrogen requirements by organic manures (FYM, RSC, DTR and MTR) according to their nitrogen content. The control vines received the nitrogen requirements as mineral alone.

The results showed that all combinations from organic nitrogen (sources + doses) with mineral nitrogen doses increased bud burst \%, bud fertility $\%$, fruiting coefficient, number of clusters per vine, cluster weight and yield/vine. In addition, SSC $\%$ was increased and total acidity was reduced in the berries juice. The data also indicated an improvement of rate of wood maturity, weight of pruning wood per vine and total carbohydrates in the canes as compared with using mineral nitrogen alone. The superiority of this investigation was for DTR at 40 and 60 units +40 and 20 units of mineral nitrogen per fed which gave the best results.
\end{abstract}

\section{INTRODUCTION}

Grape (Vitis vinifera, L.) is considered the first fruit crop in both area and production allover the world. In Egypt it is the second major fruit crop after citrus. Vineyards have increased in the last few years especially in the newly reclaimed lands. The total area of grape reached about 159929 feddans producing about 1275288 tons according to the lastest statistics of the Ministry of Agriculture (2004).

Thompson seedless grape is the most important table grape variety grown in Egypt. It is used as a fresh table grape, making raisins and for export. Grape fertilization is one of the important agricultural practices for increasing crop yield, especially the nitrogenous fertilization. The role of nitrogen in crop production has been a major concern of the viticulturists. The efficiency of chemical $\mathrm{N}$ fertilizer particularly under field conditions and surface irrigation rarely exceeds $50 \%$ and is usually about 30-40\% (Englestad \& Russel, 1975 and Sahrawat, 1979). This low efficiency may be due to losses of nitrogen from soil by leaching of nitrate and nitrite, or reduction of nitrate resulting in the formation of nitrogen gas that lost by volatilization (Goring, 1962). 
Grape growers use some mineral fertilizers such as ammonium sulphate or nitrate and urea as a sources of nitrogen because of their high content of $\mathrm{N}$ as well as their high solubility. Application of these chemical fertilizers to the soil causes some problems especially when unbalanced program of practiced was carried out. It is well known that the nitrogenous fertilizers are lost via nitrate reduction, denitrification and ammonia volatilization. Moreover, some nitrogenous fertilizer can be leached to the under groundwater causing environmental pollution (Attia, 1990; Sorial \& Abd El-Fatah, 1998 and Zaghloul, 2002).

Due to the limited amounts of organic manures applied to the vineyards in Egypt, many trials have to be carried out to find out the possibility of using some new natural materials to improve the soil fertility and productivity of grapes. In addition, it was though fruitful to depress the pollution occurring under Egyptian environmental conditions due to the exaggeration in the application of chemical and mineral fertilizers.

This investigation was carried out to throw some light on using Farmyard manure (FYM), rice straw compost (RSC), Damitta town refuses (DTR) and Mansoura town refuses (MTR) as organic nitrogen sources in combination with mineral nitrogen source to reduce mineral nitrogenous fertilizer for Thompson seedless grapevines. The final goal is to produce a healthy product less pollinated with chemical fertilizers, as well as suitable for export with lower cost by involving organic fertilizers in the manuring program of grapes.

\section{MATERIALS AND METHODS}

This investigation was carried out during three successive seasons of 2003, 2004 and 2005 at a private vineyard at EL-Taweela Village near Mansoura City, Dakahlia Governorate. The vines were of Thompson Seedless cultivar. At the start of the experiment the vines were fifteen years old, planted in a clay soil at $1.5 \times 2.5$ meters. All the experimental vines were pruned to 5 canes with 12 eyes each along with 5 renewal spurs, in all 70 buds were left per vine.

The vines were trained on three wires system and cane pruned. Some physical and chemical characteristics of the experimental site were determined before the application of the fertilization treatments according to the method outlined by Piper, (1950) and Black (1965). Soil samples were taken from three layers at depths $0-30,30-60$ and $60-90 \mathrm{~cm}$ and the result of the analysis is presented in Table (1). The experiment consists of 13 treatments arranged as a factorial experiment with two factors (application sources of organic and doses of organic plus mineral) in a complete randomized block design, each treatment include three replicates of three uniform vines. Thus, the total number of vines used in this study was 117 .

Four types of organic manures were used namely, farmyard manure (FYM), Rise straw compost (RSC), Damietta town refuses (DTR) and Mansoura town refuses (MTR). The control vines received the same $\mathrm{N}$ treatment as 80 unit/fed. as recommended by the Ministry of Agriculture. 
J. Agric. Sci. Mansoura Univ., 33 (10), October, 2008

Table 1: Chemical and physical analysis of the experimental soil before starting the experiment.

\begin{tabular}{|c|c|c|c|c|}
\hline \multirow{2}{*}{ Soil properties } & \multicolumn{4}{|c|}{ Soil depth (cm) } \\
\hline & $0-30$ & $30-60$ & $60-90$ & $0-90$ \\
\hline \multicolumn{5}{|c|}{ Physical analysis } \\
\hline Coarse sand & 2.96 & 2.82 & 2.02 & 2.60 \\
\hline Fine sand & 15.24 & 15.38 & 15.18 & 15.27 \\
\hline Silt & 42.00 & 36.00 & 36.00 & 38.00 \\
\hline Clay & 39.80 & 45.80 & 46.80 & 44.13 \\
\hline Texture & \multicolumn{4}{|c|}{ Clay loam } \\
\hline \multicolumn{5}{|c|}{ Chemical analysis } \\
\hline $\mathrm{CaCO}_{3}$ & 2.16 & 2.43 & 1.94 & 2.18 \\
\hline Organic matter & 0.91 & 0.57 & 0.45 & 0.64 \\
\hline $\mathrm{N}(\mathrm{ppm})$ & 55 & 29 & 105 & 63.0 \\
\hline $\mathrm{P}$ (ppm) & 37 & 21 & 10.60 & 22.9 \\
\hline $\mathrm{K}$ (ppm) & 533 & 360 & 285 & 392.7 \\
\hline Fe (ppm) & 2.98 & 2.88 & 1.82 & 2.56 \\
\hline $\mathrm{Mn}(\mathrm{ppm})$ & 3.98 & 3.62 & 1.96 & 3.19 \\
\hline $\mathrm{Zn}(\mathrm{ppm})$ & 1.18 & 0.64 & 0.58 & 0.8 \\
\hline $\mathrm{Cu}$ (ppm) & 5.24 & 1.44 & 0.72 & 2.47 \\
\hline $\mathrm{pH}$ & 8.12 & 8.10 & 8.06 & 8.09 \\
\hline \multicolumn{5}{|c|}{ soluble anions (Meq/100 g soil) } \\
\hline $\mathrm{CO}_{3}^{-}$ & 0.00 & 0.00 & 0.00 & 0.00 \\
\hline $\mathrm{HCO}_{3}^{-}$ & 0.20 & 0.27 & 0.34 & 0.27 \\
\hline $\mathrm{CL}^{-}$ & 0.41 & 0.51 & 0.92 & 0.61 \\
\hline $\mathrm{SO}_{4}^{-}$ & 1.07 & 2.04 & 2.12 & 1.74 \\
\hline \multicolumn{5}{|c|}{ Soluble cations (Meq/100 g soil) } \\
\hline $\mathrm{Ca}^{+}$ & 0.80 & 1.10 & 1.10 & 1.00 \\
\hline $\mathrm{Mg}^{++}$ & 0.33 & 0.97 & 1.16 & 0.82 \\
\hline $\mathrm{Na}^{+}$ & 0.48 & 0.69 & 1.07 & 0.75 \\
\hline $\mathrm{K}^{+}$ & 0.08 & 0.06 & 0.05 & 0.06 \\
\hline
\end{tabular}

The experimental vines received different combinations of both mineral $\mathrm{N}$ and organic fertilizers from different sources as shown in Tables. Table (2) shows some characteristics of organic manure used in the present study and table (3) shows the experimental treatments.

Table 2: Analysis of the used organic fertilizers (on dry weight basis).

\begin{tabular}{|l|c|c|c|c|c|c|c|c|}
\hline \multirow{2}{*}{ Constituents } & \multicolumn{2}{c}{$\begin{array}{c}\text { Farmyard } \\
\text { manure }\end{array}$} & \multicolumn{2}{c|}{$\begin{array}{c}\text { Rice straw } \\
\text { compost }\end{array}$} & \multicolumn{2}{c|}{$\begin{array}{c}\text { Damietta town } \\
\text { refuses }\end{array}$} & \multicolumn{2}{|c|}{$\begin{array}{c}\text { Mansoura town } \\
\text { refuses }\end{array}$} \\
\cline { 2 - 9 } & $\mathbf{2 0 0 4}$ & $\mathbf{2 0 0 5}$ & $\mathbf{2 0 0 4}$ & $\mathbf{2 0 0 5}$ & $\mathbf{2 0 0 4}$ & $\mathbf{2 0 0 5}$ & $\mathbf{2 0 0 4}$ & $\mathbf{2 0 0 5}$ \\
\hline $\mathbf{0 . M} \%$ & 25.30 & 16.45 & 25.49 & 15.00 & 49.28 & 15.26 & 44.82 & 11.74 \\
\hline Total N \% & 1.03 & 0.98 & 0.96 & 0.66 & 1.07 & 0.81 & 1.05 & 0.54 \\
\hline $\mathbf{C} \%$ & 14.68 & 9.54 & 14.79 & 8.70 & 28.48 & 8.85 & 25.99 & 6.81 \\
\hline $\mathbf{C} / \mathbf{N} \%$ & $14: 1$ & $10: 1$ & $15: 1$ & $13: 1$ & $26: 1$ & $11: 1$ & $24: 1$ & $13: 1$ \\
\hline $\mathbf{P} \%$ & 0.35 & 0.28 & 0.23 & 0.37 & 0.18 & 0.41 & 0.19 & 0.23 \\
\hline $\mathbf{K} \%$ & 1.93 & 0.86 & 1.32 & 0.58 & 0.91 & 0.67 & 0.91 & 0.39 \\
\hline $\mathbf{p H}$ & 7.5 & 7.6 & 6.8 & 6.6 & 7.2 & 7.7 & 7.8 & 7.8 \\
\hline Humidity & 25.7 & 11.6 & 32.8 & 8.4 & 21.9 & 6.8 & 21.4 & 4.3 \\
\hline Fe (ppm) & 5019 & 7350 & 5486 & 6410 & 2574 & 6470 & 3281 & 6440 \\
\hline $\mathbf{M n}(\mathbf{p p m})$ & 444 & 797 & 775 & 902 & 179 & 449 & 528 & 1038 \\
\hline $\mathbf{Z n}$ (ppm) & 500 & 732 & 334 & 389 & 486 & 1235 & 549 & 1080 \\
\hline
\end{tabular}


Nitrogen from mineral $\mathrm{N}$ source was added as ammonium sulphate form $(20.6 \% \mathrm{~N})$. Ammonium sulphate was applied at three equal doses after bud burst, after fruit set and after harvest. The organic fertilizers were added at the second week of January in both seasons in holes with $50 \mathrm{~cm}$ length, 40 $\mathrm{cm}$ diameter and $50 \mathrm{~cm}$ depth at a distance of $50 \mathrm{~cm}$ from the vines trunk in the two side. All vines received the basal recommended fertilizers of $100 \mathrm{~g}$ calcium super phosphate $\left(15.5 \% \mathrm{P}_{2} \mathrm{O}_{5}\right)$ at the time of adding the organic fertilizers. Potassium sulphate $\left(48 \% \mathrm{~K}_{2} \mathrm{O}\right)$ was added at the rate of $100 \mathrm{~g}$ per vine at two equal doses after fruit set and after harvest.

The experimental vines received the normal management of pests and disease control and other agriculture practices used in the vineyard.

Table 3 : The amount of nitrogen from organic and mineral sources applied in the studied thirteen treatments.

\begin{tabular}{|c|c|c|c|c|c|c|c|c|c|}
\hline \multirow{3}{*}{ No. } & \multicolumn{5}{|c|}{ Organic fertilizers } & \multicolumn{2}{|c|}{$\begin{array}{c}\text { The amount of } N \\
\text { (g/vine) }\end{array}$} & \multirow{3}{*}{$\begin{array}{l}\text { The amount } \\
\text { of mineral } \\
\text { fertilizers as } \\
\text { ammonium } \\
\text { sulphate } \\
\text { (g/vine) }\end{array}$} & \multirow{3}{*}{$\begin{array}{c}\text { Total } \\
\text { N/fed } \\
\text { (units) }\end{array}$} \\
\hline & \multirow{2}{*}{ Type } & \multicolumn{2}{|c|}{$\begin{array}{c}\text { Amount/vine } \\
(\mathrm{kg})\end{array}$} & \multicolumn{2}{|c|}{$\mathbf{N} \%$} & \multirow{2}{*}{ organic } & \multirow{2}{*}{ mineral } & & \\
\hline & & 2004 & 2005 & 2004 & 2005 & & & & \\
\hline 1 & Control & -- & -- & -- & -- & -- & 80 & 400 & 80 \\
\hline 2 & $\begin{array}{l}\text { Farmyard } \\
\text { manure (FYM) }\end{array}$ & 2.44 & 2.28 & 1.03 & 0.98 & 20 & 60 & 300 & 80 \\
\hline 3 & $\begin{array}{l}\text { Farmyard } \\
\text { manure (FYM) }\end{array}$ & 4.88 & 4.56 & 1.03 & 0.98 & 40 & 40 & 200 & 80 \\
\hline 4 & $\begin{array}{l}\text { Farmyard } \\
\text { manure (FYM) }\end{array}$ & 7.32 & 6.84 & 1.03 & 0.98 & 60 & 20 & 100 & 80 \\
\hline 5 & $\begin{array}{lr}\text { Rice } & \text { straw } \\
\text { compost } & \text { (RSC) }\end{array}$ & 2.77 & 3.29 & 0.96 & 0.66 & 20 & 60 & 300 & 80 \\
\hline 6 & $\begin{array}{ll}\text { Rice } & \text { straw } \\
\text { compost } & \text { (RSC) }\end{array}$ & 5.54 & 6.58 & 0.96 & 0.66 & 40 & 40 & 200 & 80 \\
\hline 7 & \begin{tabular}{|lr} 
Rice r & straw \\
compost & (RSC)
\end{tabular} & 8.31 & 9.87 & 0.96 & 0.66 & 60 & 20 & 100 & 80 \\
\hline 8 & $\begin{array}{l}\text { Damietta town } \\
\text { refuses (DTR) }\end{array}$ & 2.29 & 2.64 & 1.07 & 0.81 & 20 & 60 & 300 & 80 \\
\hline 9 & $\begin{array}{l}\text { Damietta town } \\
\text { refuses (DTR) }\end{array}$ & 4.58 & 5.28 & 1.07 & 0.81 & 40 & 40 & 200 & 80 \\
\hline 10 & $\begin{array}{l}\text { Damietta town } \\
\text { refuses (DTR) }\end{array}$ & 6.87 & 7.92 & 1.07 & 0.81 & 60 & 20 & 100 & 80 \\
\hline 11 & $\begin{array}{l}\text { Mansoura town } \\
\text { refuses (MTR) }\end{array}$ & 2.31 & 3.86 & 1.05 & 0.54 & 20 & 60 & 300 & 80 \\
\hline 12 & $\begin{array}{l}\text { Mansoura town } \\
\text { refuses (MTR) }\end{array}$ & 4.62 & 7.72 & 1.05 & 0.54 & 40 & 40 & 200 & 80 \\
\hline 13 & $\begin{array}{l}\text { Mansoura town } \\
\text { refuses (MTR) }\end{array}$ & 6.93 & 11.58 & 1.05 & 0.54 & 60 & 20 & 100 & 80 \\
\hline
\end{tabular}

\section{OBSERVATIONS:}

1- Bud behaviour:

Bud burst, bud fertility and fruiting coefficient were calculated according to Omran (2000) as the following :

Number of opened buds/vine

1.1 - Bud burst $\%=\frac{-}{\text { Total number of buds left/vine }(70)}$ 
J. Agric. Sci. Mansoura Univ., 33 (10), October, 2008

$\begin{aligned} & \text { Number of clusters/vine } \\ & \text { Total number of buds left/vine }(70)\end{aligned}$
$\begin{gathered}\text { Number of clusters } \\ \text { 1.3- Fruiting coefficient } \begin{aligned} \text { M } \\ \text { Bud burst number/vine }\end{aligned}\end{gathered}$

\section{2- Mineral content in the leaf petioles :}

Leaf petioles of the leaves collected from the first fully mature leaves from the top of the growing shoots ( $6^{\text {th }}$ and $7^{\text {th }}$ leaves).

2.1- Nitrogen was determined by the micro-kieldahl method as described by Cottenie, (1982).

2.2- Phosphorus was colorimetrically determined using the chloro-stannous reduced molybdophosphoric Blue colour method, Cottenie et al. (1982).

2.3- Potassium was determined using a Flame photometer according to Cottenie, (1982).

2.4- Fe, $\mathrm{Mn}$, and $\mathrm{Zn}$ were determined spectrophotometrically using (PerkinElmer Model 2380 Atomic absorption spectrophotometer) according to instruction of manufactures and according to the method described

3- Yield : by Chapman and Pratt, (1961).

Total number of clusters per vine was recorded at harvesting, six cluster/vine were weighted and the average cluster weight was multiplied by number of clusters/vine and hence average yield/vine was calculated.

4- Chemical properties of berries :

4.1- Soluble solids content (SSC\%) was determined by using a hand refractometer.

4.2 - Total acidity percentage (expressed as $\mathrm{g}$ tartaric acid per $100 \mathrm{ml}$ of juice) by titration with $0.1 \mathrm{NaOH}$ using phenolphthalene as an indicator according to A.O.A.C. (1980).

4.3- SSC/acid ratio.

\section{5- Determination of some characteristics after harvesting :}

5.1- Rate of wood maturity : twelve shoots of the current season growth were tagged for each replicate to follow up the rate of wood maturity at $15^{\text {th }}$ October in both seasons of study. Rate of wood maturity was calculated by dividing length of the mature part by the total length of the shoot according to Rizk and Rizk, (1994).

5.2- Pruning wood weight : the weight of pruning wood was determined at winter pruning time during the seasons of study and the data were recorded as $\mathrm{kg} / \mathrm{vine}$.

5.3- Total carbohydrates in the canes: total carbohydrates was determined according to modified method of Shaffer and Hartman (1921). Total carbohydrates was calculated as $\mathrm{g} / 100 \mathrm{~g}$ dry weight.

\section{Statistical analysis :}

All data of this study were statistically analyzed according to the technique of analysis of variance (ANOVA) for the factorial experiment in 
completely randomize block design according to Snedecor and Cochran (1980). The treatment means were compared using the Newly Least Significant Differences (N.L.S.D) according to the producer outlined by Waller and Duncan (1969).

\section{RESULTS AND DISCUSSION}

1- Bud behavior :

Bud burst, bud fertility and fruiting coefficient:

Data in Table 4 revealed that DTR as organic source gave the highest values of bud burst \% as compared with other organic nitrogen sources in both seasons of study. On the other hand, there were no significant differences in bud burst \% among FYM, RSC and MTR in both seasons of study. Data also showed that RSC tend to increase bud fertility \% and fruiting coefficient as compared with FYM, DTR and MTR in the two seasons of study.

Table 4: Effect of some organic and mineral nitrogen fertilization treatments on bud burst, bud fertility and fruiting coefficient percentages.

\begin{tabular}{|c|c|c|c|c|c|c|c|c|c|c|c|c|}
\hline \multirow{2}{*}{\multicolumn{2}{|c|}{\begin{tabular}{|c|} 
Cheatments \\
\end{tabular}}} & \multicolumn{3}{|c|}{$\begin{array}{l}\text { Bud burst } \\
(\%)\end{array}$} & \multicolumn{4}{|c|}{$\begin{array}{l}\text { Bud fertility } \\
(\%)\end{array}$} & \multicolumn{4}{|c|}{$\begin{array}{c}\text { Fruiting coefficient } \\
(\%)\end{array}$} \\
\hline & & \multirow{2}{*}{\multicolumn{7}{|c|}{\begin{tabular}{|l|l|l|l|}
2004 & 2005 & 2004 & 2005 \\
\end{tabular}}} & \multicolumn{2}{|c|}{2004} & \multicolumn{2}{|c|}{2005} \\
\hline \multicolumn{12}{|c|}{ A: Organic sources of $\mathrm{N}$ : } & \\
\hline \multirow{2}{*}{\multicolumn{2}{|c|}{$\begin{array}{ll}\text { Farmyard manure } & \text { (FYM) } \\
\text { Rice straw compost } & \text { (RSC) } \\
\end{array}$}} & $64.02 \mathrm{AB}$ & 67.98 & $A B$ & |19.64 & $\mathrm{B}$ & 19.58 & B & 0.68 & $\mathrm{~B}$ & 8.89 & $\mathrm{~B}$ \\
\hline & & $63.29 \mathrm{AB}$ & 67.69 & B & 20.28 & $A$ & 20.49 & $\mathrm{AB}$ & & $\mathrm{A}$ & & $A$ \\
\hline \multicolumn{2}{|c|}{ Damitta town refuses (DTR) } & \begin{tabular}{|l|l|}
64.66 & $\mathrm{~A}$ \\
\end{tabular} & 69.90 & A & 19.43 & $\mathrm{~B}$ & 20.61 & A & 3 & $B$ & .70 & $A B$ \\
\hline \multicolumn{2}{|c|}{ Mansoura town refuses (MTR) } & \begin{tabular}{|l|l|}
62.73 & $B$ \\
\end{tabular} & 66.76 & $\mathrm{~B}$ & 19.37 & $\mathrm{~B}$ & 20.25 & $\mathrm{AB}$ & 0.88 & $A B$ & 30.23 & $\mathrm{~A}$ \\
\hline \multicolumn{2}{|c|}{ N.L.S.D. $5 \%$} & 1.75 & 2.1 & & 0.6 & & 0.9 & 98 & 1.1 & 17 & & 11 \\
\hline \multicolumn{13}{|c|}{ B: Doses of organic + mineral nitrogen (units): } \\
\hline \multirow{5}{*}{\multicolumn{2}{|c|}{\begin{tabular}{|l|}
$(0)$ organic $+(80)$ mineral \\
$(20)$ organic $+(60)$ mineral \\
$(40)$ organic $+(40)$ mineral \\
$60)$ organic $+(20)$ mineral \\
N.L.S.D. $5 \%$ \\
\end{tabular}}} & $61.67 \mathrm{C}$ & 64.07 & C & 17.57 & $\mathrm{C}$ & 18.07 & C & & C & 3.33 & C \\
\hline & & \begin{tabular}{|l|l|}
$63.39 \mathrm{BC}$ \\
\end{tabular} & & $\mathrm{C}$ & 19.92 & $\mathrm{~B}$ & & $\mathrm{~B}$ & & $A B$ & 0.28 & $\mathrm{AB}$ \\
\hline & & \begin{tabular}{|l|l|}
$64.00 \mathrm{AB}$ \\
\end{tabular} & 69.37 & $\mathrm{~B}$ & 20.75 & $\mathrm{~A}$ & 21.18 & A & 2.48 & $\mathrm{~A}$ & 31.05 & A \\
\hline & & \begin{tabular}{l|l}
65.63 & $\mathrm{~A}$ \\
\end{tabular} & 72.74 & A & 20.48 & $\mathrm{AB}$ & 1.67 & $\mathrm{~A}$ & 1.25 & $\mathrm{~B}$ & 29.49 & $\mathrm{~B}$ \\
\hline & & 1.75 & 2.1 & & 0.6 & & 0.9 & 98 & 1.1 & 17 & & 11 \\
\hline \multicolumn{13}{|c|}{ C: Interaction (AB) } \\
\hline \multirow{4}{*}{ FYM } & $(0)+(80)$ & \begin{tabular}{|l|l|}
61.67 & $\mathrm{c}$ \\
\end{tabular} & 64.07 & $\mathrm{e}$ & 17.57 & d & 18.07 & e & 28.47 & d & 28.33 & de \\
\hline & $+(60)$ & \begin{tabular}{|l|l|}
63.90 & $\mathrm{abc}$ \\
\end{tabular} & & de & 19.43 & c & 19.60 & de & & $\mathrm{cd}$ & 29.87 & $b c d \epsilon$ \\
\hline & $(40)+(40)$ & \begin{tabular}{|l|l|}
64.17 & $\mathrm{abc}$ \\
\end{tabular} & 68.57 & $\mathrm{~cd}$ & 21.43 & $a b$ & 20.00 & cde & 33.43 & & 29.27 & bcde \\
\hline & $+(20)$ & \begin{tabular}{|l|l|}
66.33 & $a$ \\
\end{tabular} & 73.73 & $a b$ & 20.13 & $c$ & 20.67 & bcd & 30.40 & $\mathrm{~cd}$ & 28.10 & \\
\hline \multirow{4}{*}{ RSC } & $+(80)$ & 61.67 & 64.07 & $\mathrm{e}$ & 17.57 & $\mathrm{~d}$ & 18.07 & $\mathrm{e}$ & 28.47 & $d$ & 28.33 & de \\
\hline & $(20)+(60)$ & \begin{tabular}{|l|l|}
62.83 & $\mathrm{bc}$ \\
\end{tabular} & 66.17 & de & 20.23 & bc & 20.33 & bcd & 32.27 & $a b c$ & 30.33 & $\mathrm{bc}$ \\
\hline & $(40)+(40)$ & \begin{tabular}{|l|l|}
63.90 & $\mathrm{abc}$ \\
\end{tabular} & 68.87 & cd & 21.90 & $a$ & 21.43 & abod & 34.30 & $a$ & 31.13 & $a b$ \\
\hline & $(60)+(20)$ & $64.77 \mathrm{abc}$ & 71.67 & bc & 21.43 & $a b$ & 22.13 & $a b$ & 33.07 & $a b$ & 31.03 & $a b c$ \\
\hline \multirow{4}{*}{ DTR } & $+(80)$ & \begin{tabular}{|l|l|}
61.67 & $\mathrm{c}$ \\
\end{tabular} & 07 & $\mathrm{e}$ & 17.57 & d & 18.07 & $\mathrm{e}$ & 28.47 & $d$ & 28.33 & $\begin{array}{ll}\text { de } \\
\end{array}$ \\
\hline & $(20)+(60)$ & $64.23 \mathrm{abc}$ & 67.13 & de & 20.00 & $\mathrm{C}$ & 20.10 & $\mathrm{~cd}$ & & $\mathrm{bc}$ & 29.90 & bcde \\
\hline & $(40)+(40)$ & \begin{tabular}{|l|l|}
$65.57 \mathrm{ab}$ \\
\end{tabular} & 72.10 & $a b c$ & 19.67 & $\mathrm{c}$ & 21.40 & $a b o d$ & 30.10 & $\mathrm{~cd}$ & 30.60 & $b c$ \\
\hline & $(60)+(20)$ & \begin{tabular}{|l|l|}
67.17 & $\mathrm{a}$ \\
\end{tabular} & 76.30 & $a$ & 20.47 & $\mathrm{bc}$ & 22.87 & $a$ & 30.50 & $\mathrm{~cd}$ & 29.97 & bcde \\
\hline \multirow{4}{*}{ MTR } & $(0)+(80)$ & \begin{tabular}{|l|l}
61.67 & $c$ \\
\end{tabular} & 64.07 & $\mathrm{e}$ & 17.57 & $d$ & 18.07 & $\mathrm{e}$ & 28.47 & $d$ & 28.33 & \begin{tabular}{|l}
$3 \mathrm{de}$ \\
\end{tabular} \\
\hline & $(20)+(60)$ & $62.60 \mathrm{bc}$ & 65.77 & de & 20.00 & c & 20.03 & cde & 31.97 & $a b c$ & 30.50 & bcd \\
\hline & $(40)+(40)$ & \begin{tabular}{|l|l|}
62.37 & $b c$ \\
\end{tabular} & 67.93 & cde & 20.00 & $\mathrm{c}$ & 21.90 & $a b c$ & 32.07 & $a b c$ & 33.20 & $a$ \\
\hline & $(60)+(20)$ & \begin{tabular}{|l|l|l|}
64.27 & $\mathrm{bcc}$ \\
\end{tabular} & 69.27 & $\mathrm{~cd}$ & 19.90 & $\mathrm{C}$ & 21.00 & abod & 31.03 & $\mathrm{bc}$ & 28.87 & 7 $\mid \mathrm{cd} \epsilon$ \\
\hline & $5 \%$ & 3.50 & 4.2 & & 1.2 & & 1.9 & & & & 2. & 21 \\
\hline
\end{tabular}


Application of 60 units organic nitrogen from any source +20 units mineral nitrogen per fed gave the highest values of bud burst \% as compared with the other treatments during the two seasons of study. Also, adding 20 , 40 or 60 units organic nitrogen $+60,40$ and 20 units mineral nitrogen per fed, respectively, gave a significant increase in bud fertility \% and fruiting coefficient as compared with 80 units mineral nitrogen alone, during the two seasons of study.

These results are in agreement with those reported by Abd El-Galil et al. (2003), who found that the vines which received organic manures combined with mineral nitrogen at different levels gave higher bud burst and bud fertility as compared with those receiving mineral nitrogen alone.

\section{Mineral content in the leaf petioles :}

Data in Tables (5 and 6) clearly showed that application of DTR gave a clear increase on $\mathrm{N}, \mathrm{P}, \mathrm{Fe}, \mathrm{Mn}$ and $\mathrm{Zn}$ in the leaf petioles as compared with the other organic sources. Potassium content in the leaf petioles was significantly increased by the application of both FYM and RSC during the two seasons of study.

Table 5: Effect of some organic and mineral nitrogen fertilization treatments on $\mathrm{N}, \mathrm{P}$ and $\mathrm{K}$ contents in leaf petioles.

\begin{tabular}{|c|c|c|c|c|c|c|c|c|c|c|c|c|c|}
\hline \multirow{2}{*}{\multicolumn{2}{|c|}{\begin{tabular}{|l|} 
Characters \\
Treatments \\
\end{tabular}}} & \multicolumn{4}{|c|}{$\mathbf{N}(\%)$} & \multicolumn{4}{|c|}{$\mathbf{P}(\%)$} & \multicolumn{4}{|c|}{$\mathbf{K}(\%)$} \\
\hline & & \multicolumn{2}{|c|}{2004} & \multicolumn{2}{|c|}{2005} & \multicolumn{2}{|c|}{2004} & \multicolumn{2}{|c|}{2005} & \multicolumn{2}{|c|}{2004} & \multicolumn{2}{|c|}{2005} \\
\hline \multicolumn{14}{|c|}{ A: Organic sources of $\mathrm{N}$ : } \\
\hline \multicolumn{2}{|c|}{ Farmyard manure } & 2.25 & $\mathrm{~B}$ & 2.22 & A & 0.27 & $\mathrm{BC}$ & 0.28 & $\mathrm{~B}$ & 1.57 & A & 1.49 & A \\
\hline \multicolumn{2}{|c|}{ Rice straw compost $\quad$ (RSC) } & 2.29 & $\mathrm{~B}$ & 2.14 & $A B$ & 0.26 & $\mathrm{C}$ & 0.29 & $\mathrm{~B}$ & 1.56 & A & 1.47 & A \\
\hline \multicolumn{2}{|c|}{ Damitta town refuses (DTR) } & 2.42 & $A$ & 2.19 & $A$ & 0.30 & A & 0.31 & A & 1.48 & $\mathrm{~B}$ & 1.43 & A \\
\hline \multicolumn{2}{|c|}{ Mansoura town refuses (MTR) } & 2.34 & $\mathrm{AB}$ & 2.08 & $\mathrm{~B}$ & .29 & $\mathrm{AB}$ & 0.31 & A & 1.46 & $\mathrm{~B}$ & 1.42 & A \\
\hline \multicolumn{2}{|c|}{ N.L.S.D. $5 \%$} & \multicolumn{2}{|c|}{0.10} & \multicolumn{2}{|c|}{0.09} & \multicolumn{2}{|c|}{0.02} & \multicolumn{2}{|c|}{0.02} & \multicolumn{2}{|c|}{0.08} & \multicolumn{2}{|c|}{ N.S } \\
\hline \multicolumn{14}{|c|}{ B: Doses of organic + mineral nitrogen (units): } \\
\hline \multicolumn{2}{|c|}{ (0) organic $+(80)$ mineral } & 1.79 & $\mathrm{D}$ & \begin{tabular}{|l|l|}
1.71 \\
\end{tabular} & $\mathrm{D}$ & 0.20 & $\mathrm{D}$ & 0.22 & $\mathrm{D}$ & 1.26 & $\mathrm{D}$ & 1.29 & $\mathrm{D}$ \\
\hline \multicolumn{2}{|c|}{ (20) organic + (60) mineral } & 2.17 & $\mathrm{C}$ & 2.08 & $\mathrm{C}$ & 0.24 & $\mathrm{C}$ & 0.25 & $\mathrm{C}$ & 1.46 & $\mathrm{C}$ & 1.39 & $\mathrm{C}$ \\
\hline \multicolumn{2}{|c|}{ (40) organic + (40) mineral } & 2.56 & $\mathrm{~B}$ & 2.35 & $\mathrm{~B}$ & 0.32 & $\mathrm{~B}$ & 0.34 & $\mathrm{~B}$ & 1.64 & $\mathrm{~B}$ & 1.52 & $\mathrm{~B}$ \\
\hline (60) organic $+(2$ & 20) mineral & 2.77 & A & 2.49 & $\mathrm{~A}$ & 0.35 & A & 0.38 & A & 1.72 & A & 1.61 & $\mathrm{~A}$ \\
\hline N.L.S.D. $5 \%$ & & 0.1 & & 0.0 & & 0.0 & & 0.0 & & 0.0 & & 0.0 & \\
\hline & & & & tera & & AB) & & & & & & & \\
\hline & $(0)+(80)$ & 1.79 & $g$ & 1.71 & $g$ & 0.20 & g & 0.22 & $f$ & 1.26 & j & 1.29 & $\mathrm{i}$ \\
\hline & $(20)+(60)$ & 1.96 & $g$ & 2.13 & de & 0.23 & $\mathrm{fg}$ & 0.24 & $f$ & 1.49 & $\mathrm{~h}$ & 1.43 & $\mathrm{fg}$ \\
\hline & $(40)+(40)$ & 2.41 & cde & 2.41 & $\mathrm{bc}$ & 0.29 & de & 0.32 & $\mathrm{~d}$ & 1.73 & $\mathrm{bc}$ & 1.56 & $\mathrm{C}$ \\
\hline & $(60)+(20)$ & 2.83 & $a$ & 2.63 & $a$ & 0.34 & $\mathrm{bc}$ & 0.35 & bcd & 1.80 & $\mathrm{a}$ & 1.66 & $a$ \\
\hline & $(0)+(80)$ & 1.79 & $g$ & 1.71 & $g$ & 0.20 & $\mathrm{~g}$ & 0.22 & $f$ & 1.26 & j & 1.29 & $\mathrm{i}$ \\
\hline & $(20)+(60)$ & 2.20 & $f$ & 2.06 & ef & 0.22 & $g$ & 0.24 & $f$ & 1.52 & $\mathrm{~h}$ & 1.42 & $g$ \\
\hline InSC & $(40)+(40)$ & 2.47 & $\mathrm{~cd}$ & 2.37 & $\mathrm{bc}$ & 0.28 & de & 0.33 & $\mathrm{~cd}$ & 1.70 & $\mathrm{~cd}$ & 1.53 & cde \\
\hline & $(60)+(20)$ & 2.69 & $a b$ & 2.44 & $\mathrm{bc}$ & 0.31 & $\mathrm{~cd}$ & 0.38 & $a b$ & 1.77 & $a b$ & 1.63 & $a b$ \\
\hline & $(0)+(80)$ & 1.79 & $\mathrm{~g}$ & 1.71 & $g$ & 0.20 & $g$ & 0.22 & $f$ & 1.26 & J & 1.29 & $\mathrm{i}$ \\
\hline & $(20)+(60)$ & 2.28 & def & 2.18 & de & 0.26 & ef & 0.25 & ef & 1.40 & $\mathrm{i}$ & 1.35 & $\mathrm{~h}$ \\
\hline Din & $(40)+(40)$ & 2.76 & $a b$ & 2.36 & $\mathrm{bc}$ & 0.35 & $a b$ & 0.36 & $\mathrm{bc}$ & 1.59 & $\mathrm{fg}$ & 1.50 & de \\
\hline & $(60)+(20)$ & 2.85 & $\mathrm{a}$ & 2.50 & $a b$ & 0.38 & $a$ & 0.40 & $\mathrm{a}$ & 1.66 & de & 1.58 & $\mathrm{bc}$ \\
\hline & \begin{tabular}{|l}
$(0)+(80)$ \\
\end{tabular} & 1.79 & $g$ & 1.71 & $\mathrm{~g}$ & 0.20 & $\mathrm{~g}$ & 0.22 & $f$ & 1.26 & $j$ & 1.29 & $\mathrm{i}$ \\
\hline & $(20)+(60)$ & 2.25 & ef & 1.95 & $f$ & 0.24 & $\mathrm{fg}$ & 0.28 & $\mathrm{e}$ & 1.41 & $i$ & 1.35 & $\mathrm{~h}$ \\
\hline & $(40)+(40)$ & 2.59 & $\mathrm{bc}$ & 2.28 & $\mathrm{~cd}$ & 0.34 & $\mathrm{bc}$ & 0.34 & $\mathrm{~cd}$ & 1.54 & gh & 1.48 & ef \\
\hline & $(60)+(20)$ & 2.73 & $a b$ & 2.39 & $\mathrm{bc}$ & 0.36 & $a b$ & 0.38 & $a b$ & 1.63 & ef & 1.54 & $\mathrm{~cd}$ \\
\hline N.L.S.D. $5 \%$ & & 0.1 & & 0.1 & & 0.0 & & 0.0 & & 0.0 & & 0.0 & \\
\hline
\end{tabular}


Table 6: Effect of some organic and mineral nitrogen fertilization treatments on $\mathrm{Fe}, \mathrm{Mn}$ and $\mathrm{Zn}$ contents in leaf petioles.

\begin{tabular}{|c|c|c|c|c|c|c|c|c|c|c|c|c|c|}
\hline \multirow{2}{*}{\multicolumn{2}{|c|}{\begin{tabular}{|l|} 
Characters \\
Treatments \\
\end{tabular}}} & \multicolumn{4}{|c|}{$\mathrm{Fe}$ (ppm) } & \multicolumn{4}{|c|}{ Mn (ppm) } & \multicolumn{4}{|c|}{ Zn (ppm) } \\
\hline & & \multicolumn{2}{|c|}{2004} & \multicolumn{2}{|c|}{2005} & \multicolumn{2}{|c|}{2004} & \multicolumn{2}{|c|}{2005} & \multicolumn{2}{|c|}{2004} & \multicolumn{2}{|c|}{2005} \\
\hline \multicolumn{14}{|c|}{ A: Organic sources of $\mathrm{N}:$} \\
\hline $\begin{array}{l}\text { Farm) } \\
\text { (FYM) }\end{array}$ & ard manure & 121.08 & BC & 116.50 & B & 32.92 & B & 37.08 & $A B$ & 43.08 & $A B$ & 46.33 & B \\
\hline $\begin{array}{l}\text { Rice } \\
\text { comp }\end{array}$ & $\begin{array}{lr} & \text { straw } \\
\text { st } & \text { (RSC) } \\
\end{array}$ & 118.00 & C & 117.92 & $A B$ & 31.42 & B & 36.08 & B & 40.92 & B & 48.25 & $A B$ \\
\hline $\begin{array}{l}\text { Damit } \\
\text { refuse }\end{array}$ & $\begin{array}{lr}\text { a } & \text { town } \\
\text { s (DTR) }\end{array}$ & 127.17 & A & 120.92 & A & 36.00 & A & 39.25 & A & 45.33 & A & 50.33 & A \\
\hline $\begin{array}{l}\text { Mans } \\
\text { refuse }\end{array}$ & $\begin{array}{l}\text { ura town } \\
\text { s (MTR) }\end{array}$ & 124.92 & $A B$ & 120.08 & A & 33.92 & $A B$ & 37.75 & $A B$ & 45.17 & A & 50.17 & A \\
\hline N.L.S & D. $5 \%$ & 4.8 & & 3.2 & & 2.6 & & 3.0 & & 3.26 & & 2. & 22 \\
\hline \multicolumn{14}{|c|}{ B: Doses of organic + mineral nitrogen (units): } \\
\hline $\begin{array}{r}(0) c \\
\text { miner } \\
\end{array}$ & ganic $+(80)$ & 111.33 & D & 104.33 & D & 24.00 & D & 27.00 & D & 34.00 & D & 39.00 & D \\
\hline $\begin{array}{l}(20) \mathrm{c} \\
\text { miner }\end{array}$ & anic + (60) & 118.00 & C & 115.33 & C & 29.00 & C & 33.00 & C & 39.08 & C & 44.25 & C \\
\hline $\begin{array}{l}(40) \mathrm{c} \\
\text { miner }\end{array}$ & anic + (40) & 127.58 & B & 124.75 & B & 37.25 & B & 42.50 & B & 46.92 & B & 52.58 & B \\
\hline $\begin{array}{l}(60) \mathrm{c} \\
\text { miner }\end{array}$ & anic $+(20)$ & 134.25 & A & 131.00 & A & 44.00 & A & 47.67 & A & 54.50 & A & 59.25 & A \\
\hline N.L.S & D. $5 \%$ & 4.8 & & 3.2 & & 2.6 & & 3.0 & & 3.26 & & 2.5 & 32 \\
\hline \multicolumn{14}{|c|}{ C: Interaction (AB) } \\
\hline \multirow{4}{*}{ FYM } & $(0)+(80)$ & 111.33 & $\mathrm{~h}$ & 104.33 & $\mathrm{~h}$ & 24.00 & $g$ & 27.00 & $f$ & 34.00 & $\mathrm{~h}$ & 39.00 & $\mathrm{~h}$ \\
\hline & $(20)+(60)$ & 116.00 & fgh & 112.67 & g & 28.33 & $\mathrm{fg}$ & 32.00 & ef & 9.00 & $\mathrm{gh}$ & 43.00 & fgh \\
\hline & $(40)+(40)$ & 126.00 & cde & 122.00 & def & 37.00 & de & 42.00 & $\mathrm{bc}$ & 45.67 & def & 48.67 & def \\
\hline & $(60)+(20)$ & 131.00 & $\mathrm{bc}$ & 127.00 & bcd & 42.33 & $a b c$ & 47.33 & $a b$ & 53.67 & $a b c$ & 54.67 & $\mathrm{bc}$ \\
\hline \multirow{4}{*}{ ISC } & $(0)+(80)$ & 111.33 & $\mathrm{~h}$ & 104.33 & $\mathrm{~h}$ & 24.00 & $g$ & 27.00 & $f$ & 34.00 & $\mathrm{~h}$ & 39.00 & $\mathrm{~h}$ \\
\hline & $(20)+(60)$ & 114.67 & gh & 114.67 & $g$ & 27.00 & $\mathrm{fg}$ & 33.00 & ef & 37.00 & gh & 41.33 & gh \\
\hline & $(40)+(40)$ & 121.33 & def & 124.00 & cde & 34.00 & $\mathrm{e}$ & 40.00 & $\mathrm{~cd}$ & 42.67 & efg & 51.67 & cde \\
\hline & $(60)+(20)$ & 124.67 & cdef & 128.67 & bc & 40.67 & $\mathrm{~cd}$ & 44.33 & $\mathrm{bc}$ & 50.00 & bcd & 61.00 & $\mathrm{a}$ \\
\hline \multirow{4}{*}{ DTR } & $(0)+(80)$ & 111.33 & $\mathrm{~h}$ & 104.33 & $\mathrm{~h}$ & 24.00 & $g$ & 27.00 & $f$ & 34.00 & $\mathrm{~h}$ & 39.00 & $\mathrm{~h}$ \\
\hline & $(20)+(60)$ & 120.33 & efgh & 116.33 & fg & 32.00 & ef & 33.00 & ef & 40.00 & fgh & 45.33 & $\mathrm{fg}$ \\
\hline & $(40)+(40)$ & 133.00 & $\mathrm{bc}$ & 127.00 & bcd & 41.00 & bcd & 46.00 & $a b c$ & 49.00 & cde & 56.00 & $a b c$ \\
\hline & $(60)+(20)$ & 144.00 & a & 136.00 & a & 47.00 & a & 51.00 & a & 58.33 & $\mathrm{a}$ & 61.00 & a \\
\hline \multirow{4}{*}{ MTR } & $(0)+(80)$ & 111.33 & $\mathrm{~h}$ & 104.33 & $\mathrm{~h}$ & 24.00 & g & 27.00 & $f$ & 34.00 & $\mathrm{~h}$ & 39.00 & $\mathrm{~h}$ \\
\hline & $(20)+(60)$ & 121.00 & defg & 117.67 & efg & 28.67 & $\mathrm{fg}$ & 34.00 & de & 40.33 & fgh & 47.33 & ef \\
\hline & $(40)+(40)$ & 130.00 & bcd & 126.00 & bcd & 37.00 & de & 42.00 & $\mathrm{bc}$ & 50.33 & bcd & 54.00 & $\mathrm{~cd}$ \\
\hline & $(60)+(20)$ & 137.33 & $a b$ & 132.33 & $a b$ & 46.00 & $a b$ & 48.00 & $a b$ & 56.00 & $a b$ & 60.33 & $a b$ \\
\hline \multicolumn{2}{|c|}{ N.L.S.D. $5 \%$} & \multicolumn{2}{|c|}{9.63} & \multicolumn{2}{|c|}{6.49} & \multicolumn{2}{|c|}{5.26} & \multicolumn{2}{|c|}{6.05} & \multicolumn{2}{|c|}{6.52} & \multicolumn{2}{|c|}{5.84} \\
\hline
\end{tabular}

Adding 60 units organic nitrogen from any sources +20 units mineral nitrogen per fed gave the highest values of $\mathrm{N}, \mathrm{P}, \mathrm{K}, \mathrm{Fe}, \mathrm{Mn}$ and $\mathrm{Zn}$ content in the leaf petioles as compared with the other treatments during the two seasons of study especially when the whole amount ( 80 units of mineral nitrogen per fed) was applied solely.

The obtained results indicated that mineral content of the leaf petioles was improved by the application of organic fertilizers along with mineral nitrogen. These results are confirmed by the findings of Ezz (1999) on Thompson seedless, Harhash and Abdel-Nasser (2000) on Flame Seedless, Abd EL-Galil et al. (2003) on King Ruby and Abdel Hameed and Rabeea (2005) on Superior grapevines. They reported that supplying the 
vines with organic nitrogen in combination with mineral nitrogen significantly increased nitrogen, phosphorus, potassium, iron, manganese and zinc content on the leaves as compared with supplying them totally as mineral nitrogen source.

The improving effect of organic manures on leaf content of nitrogen , phosphorus and potassium might be attributed to their influence on increasing the organic matter in the soil (Nijjar, 1985).

The increase in the micronutrients represented in iron, manganese and zinc is due to the constituent of both the artificial organic fertilizers and also to the effect of organic matter which makes the micro nutrients more efficient to be easily absorbed by the plant (Ezz, 1999). Organic manures is an important source of macro and micro nutrients. Moreover, adding organic manure as fertilizer lead to decreasing soil $\mathrm{pH}$ which results in increasing solubility of nutrients and nutrients availability to uptake with the plants (Abou Taleb, 2004).

The increases in macro- and micro- nutrients content may be attributed to decomposition of organic compounds by microorganisms and subsequent release of their nutrient elements especially when using organic compounds, which contain high amounts of nutrient elements (Harhash and Abd EL-Nasser, 2000). The decomposition of organic materials produced some organic acids such as fulvic acid and carbonic acid which caused lower soil $\mathrm{pH}$ values and consequently increased the availability and consequently the availability in soil (Alexander, 1977 and Alawi et al., 1980).

\section{Yield and its components :}

Data presented in Table 7 showed that application of RSC gave the highest number of clusters/vine while DTR recorded a significant increase in cluster weight and yield per vine during the two seasons of study as compared with other organic nitrogen sources.

Adding 40 and 60 units organic nitrogen from any source +40 and 20 units mineral nitrogen/fed gave the highest significant increase on cluster number, cluster weight and yield/ vine as compared with the lower units of organic nitrogen plus higher units of mineral nitrogen during the two seasons of study.

The beneficial effects of using organic manures along with mineral nitrogen which caused in increasing the yield of Thompson seedless grapes in this study are in agreements with those reported by EL-Morsy (1997), Ezz (1999) on Thompson seedless, Harhash and Abd EL-Nasser (2000), Kassem and Marzouk (2002) and Abd EL-Hady et al. (2003) on Flame seedless grapes and Abd EL-Galil et al. (2003) on Ruby seedless. They found that the number of cluster/vine, cluster weight and yield per vine were increased by increasing the organic manure doses as compared with vines receiving only mineral fertilizer without applying any organic sources.

The obtained results could be explained on the bases that organic fertilizers contains high amount of available nutrient elements and humus compounds which improve soil aggregation and physical and chemical properties of soil which enhance trees growth and hence increase their yield (Omran et al., 1998). 
The positive action of the organic fertilizers on improving the yield and fruit weight could be mainly due to their effect in providing the trees with their requirement from different nutrients at longer time as well as their effect on facilitating the availability of nutrients in the soil for uptake by plants which surely reflected on enhancing the nutritional status of the trees in favour of yield and cluster weight (Nijjar, 1985).

Table 7: Effect of some organic and mineral nitrogen fertilization treatments on number of cluster, cluster weight $(\mathrm{g})$ and yield/vine $(\mathrm{kg})$.

\begin{tabular}{|c|c|c|c|c|c|c|c|c|c|c|c|c|}
\hline \multirow{2}{*}{ Characters } & \multicolumn{4}{|c|}{ Number of cluster/vine } & \multicolumn{4}{|c|}{ Cluster weight (g) } & \multicolumn{4}{|c|}{ Yield/vine (kg) } \\
\hline & \multicolumn{2}{|c|}{2004} & \multicolumn{2}{|c|}{2005} & \multicolumn{2}{|c|}{2004} & \multicolumn{2}{|c|}{2005} & \multicolumn{2}{|c|}{2004} & \multicolumn{2}{|c|}{2005} \\
\hline \multicolumn{13}{|c|}{ A: Organic sources of $\mathrm{N}$ : } \\
\hline $\begin{array}{l}\text { Farmyard manure } \\
\text { (FYM) }\end{array}$ & 13.75 & B & 13.71 & B & 432.17 & B & 423.42 & $A$ & 5.95 & $\mathrm{BC}$ & 5.82 & $B$ \\
\hline $\begin{array}{l}\text { Rice straw compost } \\
(\mathrm{RSC})\end{array}$ & 14.20 & A & 14.35 & $A B$ & 432.58 & B & 418.00 & B & 6.16 & $A B$ & 6.02 & $A B$ \\
\hline $\begin{array}{l}\text { Damitta town refuses } \\
\text { (DTR) }\end{array}$ & 13.60 & B & 14.54 & A & 467.75 & A & 423.00 & A & 6.38 & $A$ & 6.18 & A \\
\hline $\begin{array}{ll}\text { Mansoura } & \text { town } \\
\text { refuses (MTR) } & \\
\end{array}$ & 13.56 & B & 14.18 & $A B$ & 429.00 & B & 416.00 & B & 5.83 & C & 5.92 & $B$ \\
\hline N.L.S.D. $5 \%$ & 0.4 & & 0.8 & & 6.37 & & 4.15 & & 0.2 & & 0.2 & \\
\hline \multicolumn{13}{|c|}{ B: Doses of organic + mineral nitrogen (units): } \\
\hline $\begin{array}{l}(0) \text { organic }+(80) \\
\text { mineral }\end{array}$ & 12.30 & C & 12.67 & $\mathrm{C}$ & 407.67 & $\mathrm{D}$ & 371.67 & D & 5.01 & $\mathrm{D}$ & 4.69 & D \\
\hline $\begin{array}{l}(20) \text { organic }+(60) \\
\text { mineral }\end{array}$ & 13.94 & B & 14.01 & B & 423.25 & C & 416.25 & $\mathrm{C}$ & 5.90 & $\mathrm{C}$ & 5.83 & C \\
\hline $\begin{array}{l}(40) \text { organic }+(40) \\
\text { mineral }\end{array}$ & 14.53 & A & 14.94 & A & 449.50 & B & 438.42 & B & 6.51 & B & 6.54 & B \\
\hline $\begin{array}{l}(60) \text { organic }+(20) \\
\text { mineral }\end{array}$ & 14.34 & $A B$ & 15.17 & A & 481.08 & A & 454.08 & A & 6.89 & A & 6.88 & A \\
\hline N.L.S.D. $5 \%$ & 0.4 & & 0.8 & & 6.37 & & 4.15 & & 0.2 & & 0.2 & \\
\hline \multicolumn{13}{|c|}{ C: Interaction (AB) } \\
\hline$(0)+(80)$ & 12.30 & $d$ & 12.67 & $\mathrm{~d}$ & 407.67 & $g$ & 371.67 & $f$ & 5.01 & $f$ & 4.69 & $f$ \\
\hline$(20)+(60)$ & 13.60 & $\mathrm{C}$ & 13.70 & $\mathrm{~cd}$ & 427.00 & ef & 409.67 & $\mathrm{e}$ & 5.81 & $\mathrm{e}$ & 5.60 & e \\
\hline$(40)+(40)$ & 15.00 & $a b$ & 14.00 & bcd & 421.00 & $f$ & 441.67 & $\mathrm{C}$ & 6.32 & cde & 6.19 & $\mathrm{~cd}$ \\
\hline$(60)+(20)$ & 14.10 & $\mathrm{C}$ & 14.47 & $a b c$ & 473.00 & $\mathrm{C}$ & 470.67 & $a$ & 6.67 & bc & 6.80 & $a b$ \\
\hline$(0)+(80)$ & 12.30 & $d$ & 12.67 & $\mathrm{~d}$ & 407.67 & $\mathrm{~g}$ & 371.67 & $f$ & 5.01 & $f$ & 4.69 & $f$ \\
\hline$(20)+(60)$ & 14.17 & $\mathrm{bc}$ & 14.23 & bcd & 417.00 & $f g$ & 414.67 & $\mathrm{e}$ & 5.90 & de & 5.90 & de \\
\hline$(40)+(40)$ & 15.33 & $\mathrm{a}$ & 15.00 & $a b c$ & 435.00 & $\mathrm{e}$ & 439.67 & $\mathrm{C}$ & 6.67 & $\mathrm{bc}$ & 6.57 & $\mathrm{bc}$ \\
\hline$(60)+(20)$ & 15.00 & $a b$ & 15.50 & $a b$ & 470.67 & C & 446.00 & $\mathrm{bc}$ & 7.04 & $a b$ & 6.91 & $a b$ \\
\hline$(0)+(80)$ & 12.30 & $d$ & 12.67 & $d$ & 407.67 & g & 371.67 & $f$ & 5.01 & $f$ & 4.69 & $f$ \\
\hline$(20)+(60)$ & 14.00 & $\mathrm{C}$ & 14.07 & bcd & 434.00 & $\mathrm{e}$ & 425.00 & $d$ & 6.07 & de & 5.98 & de \\
\hline$(40)+(40)$ & 13.77 & $\mathrm{C}$ & 15.43 & $a b$ & 505.33 & $\mathrm{~b}$ & 441.67 & C & 6.94 & $\mathrm{~b}$ & 6.80 & $a b$ \\
\hline$(60)+(20)$ & 14.33 & $\mathrm{bc}$ & 16.00 & $\mathrm{a}$ & 524.00 & $a$ & 453.67 & $b$ & 7.50 & $a$ & 7.25 & $\mathrm{a}$ \\
\hline \multirow{5}{*}{ N.L.S.D. $5 \%$} & 12.30 & $d$ & 12.67 & $\mathrm{~d}$ & 407.67 & $\mathrm{~g}$ & 371.67 & $f$ & 5.01 & $f$ & 4.69 & $f$ \\
\hline & 14.00 & C & 14.03 & bcd & 415.00 & $\mathrm{fg}$ & 415.67 & $\mathrm{e}$ & 5.81 & $\mathrm{e}$ & 5.83 & de \\
\hline & 14.00 & $\mathrm{C}$ & 15.33 & $a b$ & 436.67 & $\mathrm{e}$ & 430.67 & $d$ & 6.12 & de & 6.60 & $\mathrm{bc}$ \\
\hline & 13.93 & C & 14.70 & $a b c$ & 456.67 & $d$ & 446.00 & $\mathrm{bc}$ & 6.37 & $\mathrm{~cd}$ & 6.54 & $\mathrm{bc}$ \\
\hline & \multicolumn{2}{|c|}{0.86} & \multicolumn{2}{|c|}{1.61} & \multicolumn{2}{|c|}{12.74} & \multicolumn{2}{|c|}{8.38} & \multicolumn{2}{|c|}{0.53} & \multicolumn{2}{|c|}{0.51} \\
\hline
\end{tabular}




\section{Chemical characteristics of berries :}

Data in Table 8 showed that insignificant differences between all organic nitrogen sources on SSC\% and SSC/acid ratio, while the application of FYM gave the lowest values of total acidity in berry juice.

Increasing the doses of organic nitrogen to 60 units with decreasing the doses of mineral nitrogen to 20 units gave the highest significant increase for SSC \% and SSC/acid ratio and the lowest significant for total acidity during the two seasons of study.

Application of organic manures along with mineral nitrogen improved both physical and chemical properties of the grapes in this study. The beneficial effect of organic fertilization on fruit quality could attributed to the effect of nutrient content of the vines which accelerated the formation of carbohydrates (Ezz, 1999).

The positive influence of the organic materials on berry quality may be a result of accumulating more carbohydrates and enhancing fruit ripening (Abd EL-Hady et al. 2003).

Table 8: Effect of some organic and mineral nitrogen fertilization treatments on soluble solids content, acidity and soluble solids content / acid ratio percentages.

\begin{tabular}{|c|c|c|c|c|c|c|c|c|c|c|c|c|}
\hline \multirow{2}{*}{ Treatments } & \multirow[t]{2}{*}{ Characters } & \multicolumn{3}{|c|}{$\begin{array}{l}\text { Soluble solids } \\
\text { content (SSC \%) }\end{array}$} & \multicolumn{4}{|c|}{ Acidity (\%) } & \multicolumn{4}{|c|}{$\begin{array}{c}\text { Soluble solids } \\
\text { content (SSC)/acid } \\
\text { ratio }\end{array}$} \\
\hline & & 2004 & \multicolumn{2}{|c|}{2005} & \multicolumn{2}{|c|}{2004} & \multicolumn{2}{|c|}{2005} & \multicolumn{2}{|c|}{2004} & \multicolumn{2}{|c|}{2005} \\
\hline \multicolumn{13}{|c|}{ A: Organic sources of $N$ : } \\
\hline \multicolumn{2}{|c|}{ Farmyard manure } & $18.59 \mathrm{~A}$ & 18.79 & $\mathrm{~A}$ & 0.51 & $\mathrm{~B}$ & 0.51 & $\mathrm{~B}$ & 36.84 & A & 36.88 & A \\
\hline \multicolumn{2}{|c|}{ Rice straw compost } & $18.43 \mathrm{~A}$ & 18.51 & $A$ & 0.53 & $\mathrm{AB}$ & 0.52 & $\mathrm{AB}$ & 35.03 & A & 35.48 & A \\
\hline \multicolumn{2}{|c|}{ Damitta town refuses (DTR) } & \begin{tabular}{|l|l|}
18.38 & $A$ \\
\end{tabular} & 18.76 & A & 0.53 & $\mathrm{AB}$ & 0.51 & $\mathrm{~B}$ & 34.89 & $\mathrm{~A}$ & 36.71 & A \\
\hline \multicolumn{2}{|c|}{ Mansoura town refuses (MTR) } & \begin{tabular}{|l|l|}
18.26 & $A$ \\
\end{tabular} & 18.39 & A & 0.54 & A & 0.53 & A & 34.20 & A & 35.09 & A \\
\hline \multicolumn{2}{|c|}{ N.L.S.D. $5 \%$} & N.S. & N.S & & 0.0 & & 0.0 & & N.S & & N.S & \\
\hline \multicolumn{13}{|c|}{ B: Doses of organic + mineral nitrogen (units): } \\
\hline \multicolumn{2}{|c|}{$(0)$ organic $+(80)$ mineral } & \begin{tabular}{|l|l|}
17.60 & $\mathrm{D}$ \\
\end{tabular} & 17.80 & $\mathrm{D}$ & 0.57 & A & 0.55 & A & 30.74 & $\mathrm{C}$ & 32.63 & $\mathrm{C}$ \\
\hline \multicolumn{2}{|c|}{ (20) organic $+(60)$ mineral } & \begin{tabular}{|l|l|}
18.19 & $\mathrm{C}$ \\
\end{tabular} & 18.40 & $\mathrm{C}$ & 0.53 & $\mathrm{~B}$ & 0.53 & $\mathrm{~B}$ & 34.45 & $\mathrm{~B}$ & 35.13 & $\mathrm{~B}$ \\
\hline \multicolumn{2}{|c|}{ (40) organic + (40) mineral } & \begin{tabular}{|l|l|}
18.54 & $B$ \\
\end{tabular} & 18.82 & $\mathrm{~B}$ & 0.51 & $\mathrm{BC}$ & 0.51 & $\mathrm{C}$ & 36.31 & $\mathrm{~B}$ & 36.84 & $\mathrm{~B}$ \\
\hline \multicolumn{2}{|c|}{ (60) organic + (20) mineral } & \begin{tabular}{|l|l}
19.33 & $A$
\end{tabular} & 19.43 & $A$ & 0.49 & $\mathrm{C}$ & 0.49 & $\mathrm{D}$ & 39.45 & $A$ & 39.56 & $\mathrm{~A}$ \\
\hline \multicolumn{2}{|c|}{ N.L.S.D. $5 \%$} & 0.34 & 0.4 & & 0.0 & & 0.0 & & 2.5 & & 2.2 & \\
\hline \multicolumn{13}{|c|}{ C: Interaction (AB) } \\
\hline \multirow{4}{*}{ FYM } & $(0)+(80)$ & 17.60 & 17.80 & $\mathrm{e}$ & 0.57 & a & 0.55 & a & 30.74 & $\mathrm{C}$ & 32.63 & $d$ \\
\hline & $(20)+(60)$ & \begin{tabular}{|l|l|}
18.40 & ef \\
\end{tabular} & 18.53 & cde & 0.52 & $\mathrm{~b}$ & 0.52 & $a b c$ & 35.77 & $\mathrm{bc}$ & 35.43 & $\mathrm{~cd}$ \\
\hline & $(40)+(40)$ & 18.77 bode & .03 & abcd & 0.50 & $\mathrm{bc}$ & 0.51 & $\mathrm{bc}$ & 37.97 & $a b$ & 37.55 & $a b c$ \\
\hline & $(60)+(20)$ & \begin{tabular}{|l|l|}
19.60 & $\mathrm{a}$ \\
\end{tabular} & 19.80 & $\mathrm{a}$ & 0.46 & $\mathrm{C}$ & 0.47 & $d$ & 42.87 & $\mathrm{a}$ & 41.91 & $a$ \\
\hline \multirow{4}{*}{ RSC } & $(0)+(80)$ & \begin{tabular}{|l|l|}
17.60 & $\mathrm{~g}$ \\
\end{tabular} & 17.80 & $\mathrm{e}$ & 0.57 & $\mathrm{a}$ & 0.55 & $\mathrm{a}$ & 30.74 & $\mathrm{C}$ & 32.63 & $\mathrm{~d}$ \\
\hline & $(20)+(60)$ & \begin{tabular}{|l|l|}
18.27 & efg \\
\end{tabular} & 18.23 & de & 0.52 & $b$ & 0.53 & $a b$ & 35.05 & $\mathrm{bc}$ & 34.29 & $\mathrm{~cd}$ \\
\hline & $(40)+(40)$ & 18.50 def & 18.73 & $\mathrm{~cd}$ & 0.52 & $b$ & 0.51 & $\mathrm{bc}$ & 35.71 & $\mathrm{bc}$ & 36.53 & bcd \\
\hline & $(60)+(20)$ & \begin{tabular}{|l|l|}
19.37 & $a b$ \\
\end{tabular} & 19.27 & $a b c$ & 0.50 & $\mathrm{bc}$ & 0.50 & bcd & 38.60 & $a b$ & 38.47 & $a b c$ \\
\hline \multirow{4}{*}{ DTR } & $(0)+(80)$ & \begin{tabular}{|l|l|}
17.60 & $\mathrm{~g}$ \\
\end{tabular} & 17.80 & $\mathrm{e}$ & 0.57 & $\mathrm{a}$ & 0.55 & $\mathrm{a}$ & 30.74 & $\mathrm{C}$ & 32.63 & $\mathrm{~d}$ \\
\hline & $(20)+(60)$ & \begin{tabular}{|l|l|}
18.07 & $\mathrm{fg}$ \\
\end{tabular} & 18.60 & cde & 0.54 & $a b$ & 0.51 & $\mathrm{bc}$ & 33.71 & $\mathrm{bc}$ & 36.58 & bcd \\
\hline & $(40)+(40)$ & 18.60 cdef & 18.97 & bcd & 0.51 & $\mathrm{~b}$ & 0.51 & $\mathrm{bc}$ & 36.52 & $\mathrm{~b}$ & 37.59 & $a b c$ \\
\hline & $(60)+(20)$ & \begin{tabular}{|l|l|}
19.27 & $a b c$ \\
\end{tabular} & 19.67 & $a b$ & 0.50 & $\mathrm{bc}$ & 0.49 & $\mathrm{~cd}$ & 38.60 & $a b$ & 40.03 & $a b$ \\
\hline & $(0)+(80)$ & \begin{tabular}{|l|l|}
17.60 & $\mathrm{~g}$ \\
\end{tabular} & 17.80 & $\mathrm{e}$ & 0.57 & $\mathrm{a}$ & 0.55 & $a$ & 30.74 & $\mathrm{C}$ & 32.63 & $\mathrm{~d}$ \\
\hline & $(20)+(60)$ & \begin{tabular}{|l|l|}
18.03 & $f g$ \\
\end{tabular} & 18.23 & de & 0.54 & $a b$ & 0.53 & $a b$ & 33.28 & $\mathrm{bc}$ & 34.20 & $\mathrm{~cd}$ \\
\hline IVIIR & $(40)+(40)$ & \begin{tabular}{|l|l|}
18.30 & ef \\
\end{tabular} & 18.53 & cde & 0.52 & $\mathrm{~b}$ & 0.52 & $a b c$ & 35.06 & $\mathrm{bc}$ & 35.68 & bcd \\
\hline & $(60)+(20)$ & 19.10 abcd & 19.00 & abcd & 0.51 & $\mathrm{~b}$ & 0.50 & bcd & 37.72 & $a b$ & 37.82 & $a b c$ \\
\hline N.L.S.D. $5 \%$ & & \begin{tabular}{|l|}
0.69 \\
\end{tabular} & 0.8 & & 0.0 & & 0.0 & & 5.4 & & 4.4 & \\
\hline
\end{tabular}


The effect of organic nitrogen fertilizers acts for controlling uptake of nitrogen by the vines for a long period and advancing their maturity could give a good explanation for the improving effect of organic manure on fruit quality (Abd EL-Galil, et al., 2003). These results are in harmony with those obtained by Ezz (1999) on Thompson seedless and Abd EL-Hady et al. (2003) on Flame seedless. They all observed that application of organic nitrogen fertilizer plus mineral nitrogen generally improved physical and chemical properties of cluster and berries.

\section{5- Determination after harvesting :}

Rate of wood maturity, pruning wood weight and total carbohydrates in canes

Regarding the effect of organic nitrogen sources, the data in Table 9 showed that adding FYM and DTR as organic nitrogen sources gave the highest percentage of wood maturity, while application of DTR as organic nitrogen source gave the highest increases in pruning wood weight and total carbohydrates in the canes as compared with other organic nitrogen sources.

Table 9: Effect of some organic and mineral nitrogen fertilization treatments on rate of wood maturity, pruning wood weight (g) and total carbohydrates (\%).

\begin{tabular}{|c|c|c|c|c|c|c|c|c|c|c|c|c|}
\hline \multirow{2}{*}{\begin{tabular}{|c|} 
Characters \\
Treatments \\
\end{tabular}} & \multicolumn{4}{|c|}{$\begin{array}{c}\text { Rate of wood maturity } \\
(\%)\end{array}$} & \multicolumn{4}{|c|}{$\begin{array}{l}\text { Pruning wood } \\
\text { weight }(\mathrm{g})\end{array}$} & \multicolumn{4}{|c|}{$\begin{array}{c}\text { Total carbohydrates } \\
(\%)\end{array}$} \\
\hline & \multicolumn{2}{|c|}{2004} & \multicolumn{2}{|c|}{2005} & \multicolumn{2}{|c|}{2004} & \multicolumn{2}{|c|}{2005} & \multicolumn{2}{|c|}{2004} & \multicolumn{2}{|c|}{2005} \\
\hline \multicolumn{13}{|c|}{ A: Organic sources of $\mathrm{N}$ : } \\
\hline Farmyard manure & 85.07 & $A$ & 83.63 & $A$ & 1.007 & $\mathrm{AB}$ & 1.053 & A & 19.56 & $\mathrm{BC}$ & 19.47 & $A$ \\
\hline Rice straw compost (RSC) & & $\mathrm{AB}$ & 81.47 & $\mathrm{~B}$ & 0.995 & B & .046 & $\mathrm{AB}$ & 9.14 & $\mathrm{C}$ & 19.36 & $\mathrm{AB}$ \\
\hline $\begin{array}{lll}\text { Damitta town refuses } \\
\text { (DTR) }\end{array}$ & 83.52 & $A B$ & 84.62 & A & 11031 & A & 1.059 & A & 19.82 & $A B$ & 19.60 & A \\
\hline $\begin{array}{l}\text { Mansoura town refuses } \\
\text { (MTR) }\end{array}$ & 81.33 & B & 79.77 & B & 1.015 & $A B$ & 1.036 & B & 20.19 & A & 19.02 & B \\
\hline N.L.S.D. $5 \%$ & 2. & & 2.0 & & 0.0 & 26 & 0.0 & & 0.5 & & 0.4 & \\
\hline \multicolumn{13}{|c|}{ B: Doses of organic + mineral nitrogen (units): } \\
\hline$(0)$ organic $+(80)$ mineral & 78.00 & C & 76.03 & $\mathrm{D}$ & 0.900 & D & 0.935 & $\mathrm{D}$ & 7.38 & $\mathrm{D}$ & 7.90 & $\mathrm{D}$ \\
\hline (20) organic + (60) mineral & 81.07 & B & 81.40 & $\mathrm{C}$ & 0.987 & $\mathrm{C}$ & 015 & $\mathrm{C}$ & & $\mathrm{C}$ & 8.86 & C \\
\hline$(40)$ organic $+(40)$ mineral & 86.60 & A & 84.49 & $B$ & 1.054 & $B$ & 1.091 & $\mathrm{~B}$ & 20.79 & $B$ & 19.98 & $\mathrm{~B}$ \\
\hline$(60)$ organic $+(20)$ mineral & 87.85 & $\mathrm{~A}$ & 87.56 & A & 1.107 & A & 1.153 & A & 21.36 & $\mathrm{~A}$ & 20.70 & $\mathrm{~A}$ \\
\hline N.L.S.D. $5 \%$ & 2. & & 2.0 & & 0.0 & & 0.0 & & 0.5 & & & 43 \\
\hline \multicolumn{13}{|c|}{ C: Interaction (AB) } \\
\hline \multirow{4}{*}{ FYM } & 78.00 & $f$ & 76.03 & $\mathrm{~g}$ & 0.900 & $\mathrm{~h}$ & 0.935 & $f$ & 17.38 & $\mathrm{~g}$ & 17.90 & $\mathrm{~h}$ \\
\hline & 83.20 & bcdef & 83.23 & de & 0.980 & $\mathrm{fg}$ & 1.021 & $\bar{e}$ & 19.16 & ef & 19.10 & efg \\
\hline & 88.87 & $\mathrm{ab}$ & 85.90 & bcd & 1.050 & cde & 1.095 & d & 20.41 & $\mathrm{~cd}$ & 20.00 & bcd \\
\hline & 90.20 & $a$ & 89.37 & $a b$ & 1.099 & $a b c$ & 1.160 & $a b$ & 21.30 & $a b c$ & 20.87 & $\mathrm{a}$ \\
\hline \multirow{4}{*}{ RSC } & 78.00 & $f$ & 76.03 & $\mathrm{~g}$ & 0.900 & $\mathrm{~h}$ & 0.935 & $f$ & 17.38 & $\mathrm{~g}$ & 17.90 & $\mathrm{~h}$ \\
\hline & 81.40 & cdef & 80.83 & ef & 0.966 & $\mathrm{~g}$ & 1.013 & $\mathrm{e}$ & 18.80 & $f$ & 18.95 & $\mathrm{fg}$ \\
\hline & 85.63 & abcd & 83.03 & de & 1.028 & def & 1.085 & d & 19.81 & de & 19.82 & code \\
\hline & 89.37 & $\mathrm{a}$ & 85.97 & bcd & 1.085 & $a b c$ & 1.149 & $a b$ & 20.55 & $\mathrm{~cd}$ & 20.76 & $a b$ \\
\hline \multirow{4}{*}{ DTR } & 78.00 & $f$ & 76.03 & $\mathrm{~g}$ & 0.900 & $\mathrm{~h}$ & 0.935 & $f$ & 17.38 & $\mathrm{~g}$ & 17.90 & $\mathrm{~h}$ \\
\hline & 80.00 & def & 84.03 & cde & 1.012 & efg & 1.025 & $\mathrm{e}$ & \begin{tabular}{|l|}
19.41 \\
\end{tabular} & ef & \begin{tabular}{|l|}
18.90 \\
\end{tabular} & $\mathrm{fg}$ \\
\hline & 89.03 & $a b$ & 87.50 & $a b c$ & 1.078 & bcd & 1.105 & $\mathrm{~cd}$ & 20.83 & $\mathrm{bc}$ & 20.63 & $a b c$ \\
\hline & 87.03 & $a b c$ & 90.90 & $\mathrm{a}$ & 1.135 & $a$ & 1.172 & $a$ & 21.67 & $a b$ & 20.96 & $\mathrm{a}$ \\
\hline \multirow{5}{*}{ MTR } & 78.00 & $\mathrm{f}$ & 76.03 & $\mathrm{~g}$ & 0.900 & $\mathrm{~h}$ & 0.935 & $f$ & 17.38 & $\mathrm{~g}$ & 17.90 & $\mathrm{~h}$ \\
\hline & 79.67 & ef & 77.50 & $\mathrm{fg}$ & 0.991 & $\mathrm{fg}$ & 1.000 & $\mathrm{e}$ & 19.34 & ef & 18.50 & gh \\
\hline & 82.87 & cdef & 81.53 & ef & 1.060 & bcde & 1.080 & $d$ & 22.11 & $a$ & \begin{tabular}{|l|}
19.46 \\
\end{tabular} & def \\
\hline & 84.80 & abcde & 84.00 & \begin{tabular}{|l} 
cde \\
\end{tabular} & 1.110 & $\mathrm{ab}$ & 1.130 & $\mathrm{bc}$ & 21.92 & $a$ & 20.20 & abcd \\
\hline & & & 4.1 & & 0.0 & & & & & & & \\
\hline
\end{tabular}


Adding 60, 40 and 20 units organic nitrogen from any source $+20,40$ and 60 units mineral nitrogen, respectively, gave a significant increase of rate of wood maturity, pruning wood weight and total carbohydrates in canes as compared with 80 units mineral nitrogen alone.

These results are in line with those reported by Abd El-Hady, et al. (2003) on Flame seedless. They found that addition of organic manures in combination with mineral nitrogen fertilizer significantly increased rate of wood maturity and pruning wood weight as compared with adding mineral nitrogen alone during the two seasons of study.

This investigation suggest that replacing $50-75 \%$ of mineral nitrogen requirements for Thompson seedless grapevines by organic nitrogen in a clay soil is indispensable for improvement of the nutritional status of the vines and production of maximum yield and quality grapes. In addition organic manures are of a great value to decrease the environmental pollution which could occur by excessive chemical fertilization alone. The best organic fertilizer in this investigation was Damitta Town refuses.

\section{REFERENCES}

A.O.A.C. (1980). Association of Official Analytical. $14^{\text {th }}$ ed Published by A.O.A.C., Washington D.C., USA.

Abd El-Galil, H.A.; M.M. Elsouky and M.M. EL-Wasfy (2003). Effect of some cultivation practices on King's Ruby grapevines production under Assiut conditions. A. effect of organic manure and yeast application on growth and nutrients status as well as yield and berry quality. Assuit $\mathrm{J}$. Agric. Sci., 34(6): 173-192.

Abd El-Hady, A.M.; M.A. Aly and M.M. EL-Mogy (2003). Effect of some soil conditioners on counteracting the adverse effects of salinity on growth and fruiting of Flame seedless vines. Minia J. Agric. Res. Develop., 23(4): 699-726.

Abd El-Hameed, H.M. and M. Rabeea (2005). Adjusting the best proportion of mineral, organic and biofertilizers for Superior grapevines. Minia J. Agric. Res. Develop., 25(5): 863-882.

Abou - Taleb, S.A. (2004). Effect of cattle manure and reducing mineral fertilizer on growth, fruit quality and nutrient content of pecan trees. Annals of Agric. Sci., Moshtohor, 42(3): 1197-1214.

Alawi, B.J. ; J.L. Stroehlein ; E.A. Hanlan and F. Turner (1980). Quality of irrigation water and effects of sulphuric acid and gypsum on soil properties and sudan grass yield. Soil Sci., 129: 315-319.

Alexander, M. (1977). Introduction to Soil Microbiology. $2^{\text {nd }}$ ed., Johan Wiley and Sons Inc., New York, pp: 467.

Attia, M.A. (1990). The biochemistry of urea decomposition by soil organisms. Ph.D thesis, Fac. Agric., Minia Univ.

Black, C.A. (1965). Methods of Soil Analysis, Chemical and Microbiological Properties (part 2). Amer. Soc. Agr. Inc.

Cottenie, A.; M. Verloo; L. Kiekens; G. Relgho and Camerlynck (1982). Chemical Analysis of Plant and Soil. Lab. Anal. Agrochemistry, State Univ., Gent, Belgium. 
Chapman, H.D. and P.F. Partt (1961). Methods of Analysis for Soil, Plants and Wasters. Div. Agric. Sci., Univ. Californiia, Berkely, Pp 309.

EL-Morsy, F.M. (1997). Response of Banaty grapevines to application of organic fertilizers filter mud and sludges. Annals of Agric. Sci. Moshtohor, 35(1): 477-488.

Engelstad, O.P. and S.A. Russel (1975). Fertilizers for use under tropical condition. Adv. Agron., 27:175-208.

Ezz, Th. M. (1999). Response of Thompson seedless grapevines grown in calcareous soil to organic fertilizer "Biotreasure" and cattle manure application. J. Agric. Sci. Mansoura Univ., 24(4): 1987-1996.

Goring, C.A.L. (1962). Control nitrification by 2-chloro-6-(trichloro-methyl) pyrine. Soil Sci., 93:21-218.

Harhash, M.M. and G. Abd EL-Nasser (2000). Effect of organic manures in combination with elemental sulpher on soil physical and chemical characteristics, yield, fruit quality, leaf water contents and nutritional status of Flame seedless grapevines. J. Agric. Sci. Mansoura Univ., 25(5): 2819-2837.

Kassem, H.A. and H.A. Marzouk (2002). Effect of organic and/or mineral nitrogen fertilization on the nutritional status, yield and fruit quality of Flame seedless grapevines grown in calcareous soil. J. Adv. Agric. Res., 7(3): 117-126.

Nijjar, G.S. (1985). Nutrition of Fruit Trees. Mrs Usha Raj Kumar, Kalyani, New Delhi, pp: $10-20$

Omran, M.S. ; B.Y. El-Koumey and L.M.S. Shokar (1998). Effect of organic and inorganic fertilizers on navel organic yield (quality and quantity). Zagazig J. Agric. Res., 25 (1): 163-174.

Omran, Y.A.M. (2000) Studies on histophysiologican effects of dormex and yeast application on bud fertility, vegetative growth and yield of Roomy Red grapevines. Ph.D. Thesis, Fac. of Agric., Assuit Univ.

Piper, C.S. (1950). Soil and Plant Analysis. Inter-Science Publisher, New York, pp. 42-110.

Rizk, I.A. and N.A. Rizk (1994). Effect of Dormex on bud behaviour, yield and rate of wood maturity of Thompson Seedless grapevines. Egypt. J. Appl. Sci., 9:525-542.

Schaffer, P.A. and A.F. Hartman (1921). The idooometric determination of copper and its use in sugar analysis. J. Bio. Chem., 45:349-364.

Sahrawat, K.L. (1979). Nitrogen losses in rice soils. Fert. News 24:38-48.

Snedecor, G. A. and W. G. Cochran (1980). Statistical Methods, $6^{\text {th }}$ ed, lowa State Univ. Press, Ames, IOWA, USA.

Sorial, M.E. and M.A. Abd EL-Fattah (1998). The possibility of using the biofertilizers as a completely substitute of NPK fertilizers in plant production. Annals of Agric. Sci. Moshtohor, 41(2): 799-817.

Waller, R.A. and D.B. Duncan (1969). A bays rule for the symmetric multiple comparison problem. J. Amer. Assoc., 64: 1484-1503.

Zaghloul, R.A. (2002). Biofertilization and organic manuring efficiency on growth and yield of potato plants (Solanum tuberosum L.). Recent Technological in Agric. Proc. of the 2nd congress vol. (1) 79-94. 


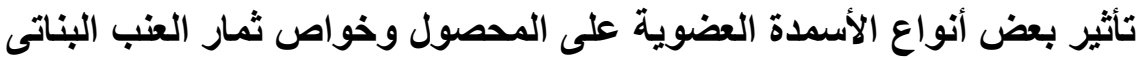

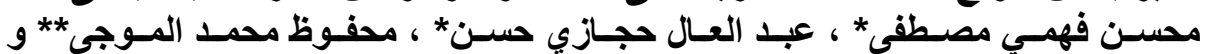

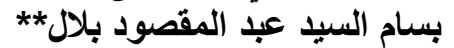
* قسم الفاكهة - كلية الزراعة المعاد - جامعة المنصورة. * معهز بحوث البساتين - مركز البحوث الزعة الزراعية.

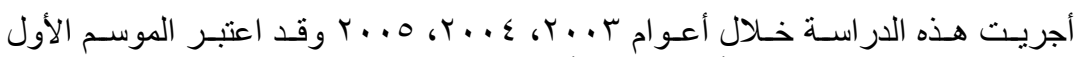

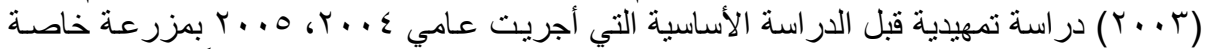

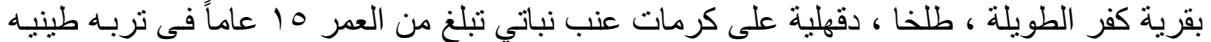

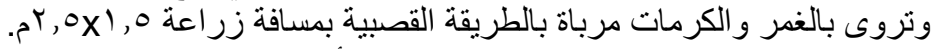

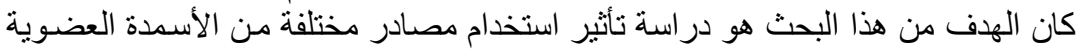

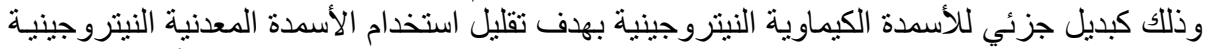

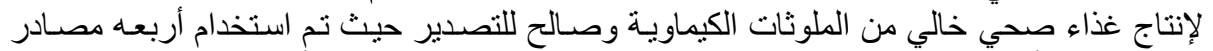

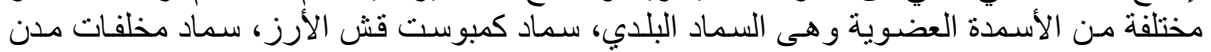

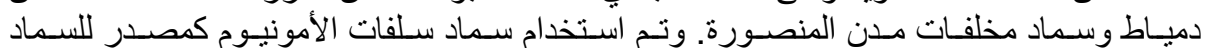

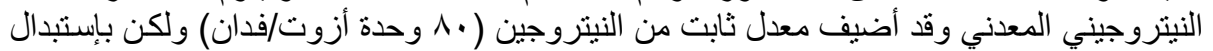

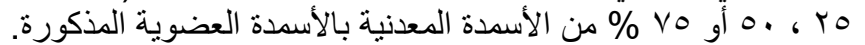

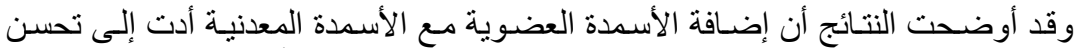

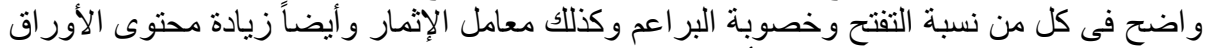

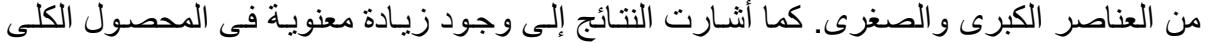

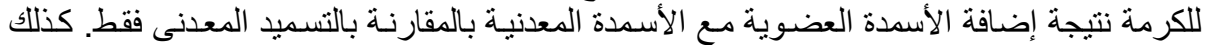

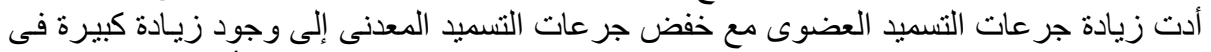

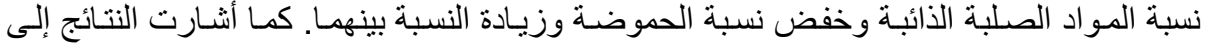

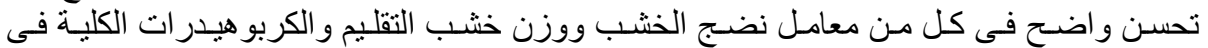

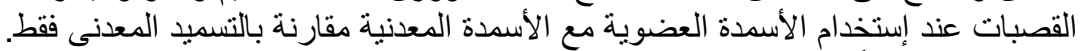

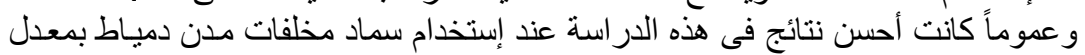

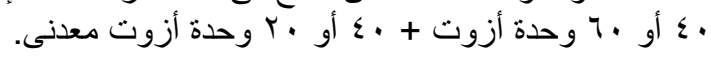

